ACCEPTED MANUSCRIPT

\title{
The role of velocity derivative skewness in understanding non- equilibrium turbulence
}

To cite this article before publication: Feng Liu et al 2020 Chinese Phys. B in press https://doi.org/10.1088/1674-1056/abbbdc

\section{Manuscript version: Accepted Manuscript}

Accepted Manuscript is "the version of the article accepted for publication including all changes made as a result of the peer review process, and which may also include the addition to the article by IOP Publishing of a header, an article ID, a cover sheet and/or an 'Accepted

Manuscript' watermark, but excluding any other editing, typesetting or other changes made by IOP Publishing and/or its licensors"

This Accepted Manuscript is $\odot 2020$ Chinese Physical Society and IOP Publishing Ltd.

During the embargo period (the 12 month period from the publication of the Version of Record of this article), the Accepted Manuscript is fully protected by copyright and cannot be reused or reposted elsewhere.

As the Version of Record of this article is going to be / has been published on a subscription basis, this Accepted Manuscript is available for reuse under a CC BY-NC-ND 3.0 licence after the 12 month embargo period.

After the embargo period, everyone is permitted to use copy and redistribute this article for non-commercial purposes only, provided that they adhere to all the terms of the licence https://creativecommons.org/licences/by-nc-nd/3.0

Although reasonable endeavours have been taken to obtain all necessary permissions from third parties to include their copyrighted content within this article, their full citation and copyright line may not be present in this Accepted Manuscript version. Before using any content from this article, please refer to the Version of Record on IOPscience once published for full citation and copyright details, as permissions will likely be required. All third party content is fully copyright protected, unless specifically stated otherwise in the figure caption in the Version of Record.

View the article online for updates and enhancements. 


\title{
The role of velocity derivative skewness in understanding non-equilibrium turbulence*
}

\author{
Feng Liu(刘锋 $)^{1,2 \dagger}$, Le Fang(方乐 $)^{2}$, and Liang Shao(邵亮 $)^{3}$ \\ ${ }^{1}$ School of Energy and Power Engineering, North University of China, \\ Taiyuan 030051, China \\ ${ }^{2}$ Laboratory of Mathematics and Physics, Ecole Centrale de Pékin, \\ Beihang University, Beijing 100191, China \\ ${ }^{3}$ Laboratoire de Mécaniques des Fluides et d'Acoustique, Ecole Centrale \\ de Lyon-Université de Lyon, Ecully 69134, France
}

September 24, 2020

\section{Abstract}

The turbulence governed by the Navier-Stokes equation is paramount in many physical processes. However, it has been considered as a challenging problem due to its inherent nonlinearity, non-equilibrium and complexity. Herein, we review the connections between the velocity derivative skewness $S_{k}$ and the non-equilibrium properties of turbulence. $S_{k}$, a reasonable candidate for describing the non-equilibrium turbulence, which varies during the non-equilibrium procedure. A lot of experimental or numerical evidences have shown that the perturbation of energy spectrum, which associated with the excitation of large scales, results in an obvious variation of $S_{k}$, and $S_{k}$ is a negative value in this rapid energy decay process. The variation of positive $S_{k}$ is closely related to the perturbation of transfer spectrum, and this corresponds to the backward energy transfer process. In addition, the skewness characterizes the production (or reduction) rate of enstrophy due to vortex stretching (or compression). Using the transport equation of turbulent energy dissipation rate and enstrophy, it is possible to establish a theoretical connection between skewness and the non-equilibrium turbulence. It is expected that this work could trigger the rapid advancement of the future studies of non-equilibrium turbulence, and also the improvement of turbulence models.

Keywords: velocity derivative skewness; non-equilibrium turbulence; turbulence model

PACS: $47.10 .-\mathrm{g} ; 47.27 .-\mathrm{i}$

\section{Introduction}

The Richardson-Kolmogorov energy cascade is an important fundamental theory for turbulence dissipation at high Reynolds numbers, which leads to a conclusion that the energy transfer in the inertial range should *Project supported by the National Natural Science Foundation of China (Grant No. 11772032), the Science Foundation of North University of China (Grant No. 11026829).

†Corresponding author. E-mail:feng.liu@nuc.edu.cn 
be balanced with dissipation, that is, an equilibrium state of turbulence. In recent ten years, a special state of turbulence which is referred to as "non-equilibrium turbulence" has received a great deal of attention of scientists ${ }^{[1,2]}$. This type of turbulence violates the equilibrium Richardson-Kolmogorov energy cascade which is acceptable in traditional turbulence theories. Numerous important advances have been made over the last decades in understanding the properties of non-equilibrium turbulence through experimental and numerical data ${ }^{[1]}$. However, the mechanism of non-equilibrium remains an unsolved problems in physics. The dissipation coefficient, defined as $C_{\epsilon}=\epsilon \mathcal{L} / \mathcal{U}^{3}$, is a quantity that has been widely used in many investigations for describing the non-equilibrium state of turbulence. Here $\epsilon$ is the turbulent energy dissipation rate, $\mathcal{U}$ is the rms of the velocity fluctuations, and $\mathcal{L}$ is the integral length scale. It is commonly believed that $C_{\epsilon}$ varies in the non-equilibrium region and bocomes approximately constant in the equilibrium region. Due to the difficulty of defining the integral scale $\mathcal{L}$ in anisotropic flows with complex geometries, $C_{\epsilon}$ has a limited scope of application ${ }^{[3]}$. Fortunately, some recent studies have shown that the skewness of longitudinal yelocity derivative, usually defined as $S_{k}=\left\langle(\partial u / \partial x)^{3}\right\rangle /\left\langle(\partial u / \partial x)^{2}\right\rangle^{3 / 2}$, plays an important role in assessing the non-equilibrium of turbulence ${ }^{[4-6]}$, with $u$ the longitudinal velocity fluctuation and $x$ the flow direction. Clearly, the obtainment of $S_{k}$ is much easier than $C_{\epsilon}$ to some extent. Indeed, $C_{\epsilon}$ can only describes the phenomenon which is related to the second-order moments ( $\epsilon$ and $\mathcal{L}$ are second-order moments), wihle $S_{k}$ represents the relation between second- and third-order moments, which is perfect in evaluating the ratio between the energy transfer and the energy dissipation. $S_{k}$ is an important small-scale quantity in turbulence since it is closely related to the fine interactions among all scales and to the production of enstrophy or energy dissipation rate. Furthermore, $S_{k}$ is valuable in turbulence modelling, such as the classical $k-\epsilon$ model.

In the past couple of decades, many studies focus on the Reynolds number (usually refers to the Taylor microscale Reynolds number $\operatorname{Re}_{\lambda}$ ) dependence of the $S_{k}{ }^{[7-16]}$, especially when the $\operatorname{Re}_{\lambda}$ is very high. However in the present work, we are rather interested in providing a review on the connections between $S_{k}$ and the non-equilibrium properties of turbulence. Recently, using grid-generated turbulence experiments, Isaza et al. found that $S_{k}$ can be used to identify the difference between the non-equilibrium near-field and the equilibrium far-field ${ }^{[5]}$. Subsequently, Hearst and Lavoie showed that the rapid changes in $S_{k}$ are related to the turbulent regions of non-equilibrium ${ }^{[4]}$, as are the results of Liu et al. in their transitional channel flows ${ }^{[17]} . S_{k}$ approachs a constant value in the equilibrium turbulence. Employing the $S_{k}$ of the resolved velocity field, Fang et al. proposed an improved Smagorinsky model to correct the non-equilibrium behavior of the traditional Smagorinsky model ${ }^{[6]}$. The recent papers ${ }^{[18,19]}$ show that the transport equation for $\epsilon$ represents, in essence, a constraint on how $S_{k}$ varies in different flows, which can be used to determine the $k-\epsilon$ model constants. This is a very important analytical tooll to undérstand the behavior of $S_{k}$ and can be extended to non-equilibrium turbulence studies.

In fact, before these studies, some previous investigations suggest that the initial conditions and the forms of turbulent flow can give rise to a change in the value of $S_{k}{ }^{[20-24]}$. This behaviour is of course understandable, since the above conditions could introduce inhomogeneity or non-stationarity in large scales. Although the study of non-equilibrium turbulence has already been reviewed in the past years, to the best of our knowledge, a specific review on the relationship between $S_{k}$ and the non-equilibrium turbulence remains scarce. The goal of this work is to broaden the understanding of non-equilibrium mechanism through the properties of $S_{k}$. This 
review is structured as follows: we present the nature and the features of $S_{k}$ in Section 2. In Section 3, we review the evolution of $S_{k}$ under the perturbation of energy spectrum and transfer spectrum respectively. Section 4 generalizes the relationship between skewness and the enstrophy, and the relationship between skewness and turbulent kinetic energy dissipation rate. Finally, in Section 5, the summary and discussion are concluded, and new perspectives, opportunities and challenges of $S_{k}$ on future non-equilibrium studies are addressed.

\section{The features of the velocity derivative skewness}

It is well known that the probability distribution function (PDF) of the turbulent velocity is Gaussian in isotropic turbulence. However, the PDF of the longitudinal velocity gradient $\partial u / \partial x$ is non-Gaussian and skews towards negative values, namely, $S_{k}$ is a negative value in turbulence. In fact $S_{k}$ can also be positive as documented by numerous investigations ${ }^{[25-27]}$, this will be elaborated in the following sections. Although $S_{k}$ is a local quantity, it implies the global balance of energy transfer over all seales ${ }^{[6]}$. The expression for $S_{k}$ in the spectral space is given by

$$
S_{k}(t)=\frac{\left\langle(\partial u / \partial x)^{3}\right\rangle}{\left\langle(\partial u / \partial x)^{2}\right\rangle^{3 / 2}}=-\frac{3 \times \sqrt{30}}{14} \frac{\int_{0}^{\infty} k^{2} T(k, t) d k}{\left[\int_{0}^{\infty} k^{2} E(k, t) d k\right]^{3 / 2}},
$$

with $k$ the wavenumber, $E(k, t)$ the energy spectrum and $T(k, t)$ the transfer spectrum. This expression is mentioned in many previous studies ${ }^{[28-30]}$. The numerator of Eq. (1) represents a flux of energy transfer and scales in the same way as the mean-square second velocity derivative ${ }^{[31]}$. The denominator of Eq. (1) represents the energy dissipation as it can be expressed as $\epsilon=2 \nu \int_{0}^{\infty} k^{2} E(k, t) d k$. This means that $S_{k}$ describes the ratio between the energy transfer and the energy dissipation, which same to that in the dissipation coefficient $C_{\epsilon}$ as summarized by Vassilicos ${ }^{[1]}$. Eq. (1) also reveals that $S_{k}$ is closely linked to the evolution of energy spectrum $E(k, t)$ and energy transfer spectrum $T(k, t)$. For non-equilibrium turbulence, the energy spectrum in the inertial range exhibit a $k^{-5 / 3}$ power-law, which have no noticeable difference with that in equilibrium turbulence ${ }^{[1]}$. However, a significantly difference between these two turbulent states is that there is a spectral spike in smallwavenumber range of non-equilibrium turbulence, which is propesed by many investigations ${ }^{[32-34]}$. Clearly, The perturbation of energy spectrum (the presence of a spectral spike) plays an important role in the dynamics of non-equilibrium turbulence. Nevertheless, a recent study suggested that the essential origin of non-equilibrium should be the perturbation of energy transfer ${ }^{[35]}$, the disscussions in this regard will be elaborated in the following sections.

Since the nonlinear energy transfer between wavenumbers is an important process in turbulent flows, a fundamental question in connection with this energy transfer is whether it is typically between neighboring wavenumbers, or between wavenumbers that are more widely separated ${ }^{[36]}$. According to Kolmogorov's equilibrium theory, the energy transfer in the inertial range is predominately local, that is, most of the energy is transferred between neighboring wavenumbers ${ }^{[37,38]}$. However, Many studies argue that the interscale energy transfer is characterized by nonlocal triad interactions as well ${ }^{[39-41]}$. One may thus expect that a relation exists between the nonlocal traid interactions of energy transfer and the non-equilibrium properties of turbulence. This calls for future studies on considering the local traid interactions and the nonlocal traid interactions of the $S_{k}$ 


\section{Evolution of the skewness in non-equilibrium turbulence}

The non-equilibrium phenomena have been observed in various turbulent flows, including homogeneous isotropic turbulence ${ }^{[42,43]}$, grid-generated turbulence ${ }^{[44-47]}$, time reversed turbulence ${ }^{[35]}$, turbulent wakes ${ }^{[48-50]}$, turbulent planar jet ${ }^{[51]}$, turbulent mixing layer ${ }^{[34]}$, planar turbulent plume ${ }^{[52]}$, turbulent boundary layer ${ }^{[53]}$ and transitional channel flows ${ }^{[17]}$. It is generally accepted that the origin of non-equilibrium might be attributed to the excitation of large scales or small scales by external forcing, or to the turbulence generating conditions which consist of different disturbances. In addition to the universal non-equilibrium law, $C_{\epsilon} \sim \mathrm{Re}_{\lambda}^{-1}$, which is confirmed by Vassilicos ${ }^{[1]}$, a recent study reveals that a new non-equilibrium law, $C_{\epsilon} \sim \operatorname{Re}_{\lambda}^{-2}$, is available in time-reversed turbulence ${ }^{[35]}$. The perturbation of energy spectrum plays a dominant role in the former, while the later is relevant to the perturbation of transfer spectrum primarily. Thus in this section, we discuss the evolution of $S_{k}$ under the perturbation of energy spectrum and transfer spectrum respectively. It is important for the following to note that the terms "universal non-equilibrium law" or "the first non-equilibrium law" are employed to represent the scaling $C_{\epsilon} \sim \operatorname{Re}_{\lambda}^{-1}$, and the terms "new non-equilibrium law" or "the second non-equilibrium law" are employed to represent the scaling $C_{\epsilon} \sim \operatorname{Re}_{\lambda}^{-2}$.

\subsection{Evolution of the skewness under the perturbation of energy spectrum}

The universal non-equilibrium law $C_{\epsilon} \sim \operatorname{Re}_{\lambda}^{-1}$ mentioned above is such that $C_{\epsilon}$ is non-constant and with the turbulence decay rapidly in the non-equilibrium region. Although the turbulent energy dissipation in nonequilibrium turbulence violate Kolmogorov's equilibrium cascade, the direction of energy flux is maintained, that is, a forward energy cascade from large to small scales. Most of the investigations believed that the origin of non-equilibrium could be connected with the perturbation of energy spectrum which is induced by the excitation of large scales or small scales in turbulence. At present, the widely studied non-equilibrium turbulence are driven by large scales, and the cases of such type are mainly discussed in this subsection. The small scales excited cases, of course, will also be covered here though it is rarely mentioned in non-equilibrium turbulence studies.

Using wind tunnel grid-generated turbulence data, Mazellier and Vassilicos showed that $C_{\epsilon}$ is directly and strongly dependent on the dimensionless number $C_{S}{ }^{\prime}$ which represents the number of large-scale eddies within an integral scale ${ }^{[47]}$. This means that $C_{\epsilon}$ changes with $C_{S}{ }^{\prime}$, and the non-equilibrium of this type is closely related to the perturbation of large scales. A similar behavior was observed in decaying and forced turbulence where $C_{\epsilon}$ depends on the energy distribution in the small-wavenumber range (i.e. large scales) and on the forcing imposed on small wavenumbers ${ }^{[54]}$. Goto and Vassilicos also reported this non-equilibrium law via direct numerical simulations (DNSs) of homogeneous isotropic turbulence ${ }^{[43]}$. Note that their turbulent cases are calculated with periodic forcing terms applied to the large scales. Subsequently, in another DNSs of freely decaying periodic turbulence, they discovered that the non-equilibrium of turbulence is always accompanied by the presence of large-scale coherent structures (with a similar length scale to $\mathcal{L}$ ), which is evidenced by the spectral spikes in the small-wavenumber range ${ }^{[32]}$. The dissipation law changes from $C_{\epsilon} \sim \operatorname{Re}_{\lambda}^{-1}$ to a constant $C_{\epsilon}$ when the spectral spikes are disappearing. Large-scale coherent structures are excited by the Kelvin-Helmholtz $(\mathrm{K}-\mathrm{H})$ instability in the turbulence generating process ${ }^{[55]}$. Meldi and Sagaut confirmed the above findings by investigating a special fast-decay homogeneous isotropic turbulence ${ }^{[33]}$. Considering a peculiar initial energy 
spectrum with a bump at the large scales, which is produced by the fractal/multiscale objects, they showed that the non-equilibrium decay regimes are resulted from the relaxation of this non-classical energy spectrum, the evolution of $C_{\epsilon}$ is consistent with the experimental results of grid-generated turbulence. The same results were obtained by Takamure et al., performing a DNS for the spatially developing shear mixing layer, they showed that the non-equilibrium law $C_{\epsilon} \sim \operatorname{Re}_{\lambda}^{-1}$ is caused by the spikes in small-wavenumber range of energy spectrum ${ }^{\text {[34] }}$. They also demonstrated this via an analysis of the probability density function (PDF) of large-scale structures. In fact, an obvious energy bump at the large scales is often observed both experimentally and numerically, as documented by Refs. [32,34,56].

What happens if the excitation is directly and strongly introduced in small scales while maintaining the large scales unchanged. Wiltsea and Glezer carried out an experimental investigation on direct excitation of the small scales in the dissipation range of a free shear flow, and they observed that this excitation can accelerate the energy cascade from the large to the small scales, which agree with the first non-equilibrium phenomenology ${ }^{[57]}$. Interestingly, a spectral peak is appeared in the small-wavenumber range during the dissipation of turbulence, similar to the results presented in large scale excitation turbulence. Indeed, this fact also recorded in their previous work ${ }^{[58]}$ and in the work of Yeung et al. ${ }^{[59]}$.

Currently, there is little systematic theory research on explaining the non-equilibrium phenomena, nevertheless, some studies are valuable for reference. Here a discussion of the results of Bos and Rubinstein on the non-equilibrium turbulence is appropriate ${ }^{[60]}$. They decompose the energy spectrum $E(k, t)$ into its equilibrium part $\bar{E}(k, t)$ and non-equilibrium part $\tilde{E}(k, t)(E(k, t)=\bar{E}(k, t)+\tilde{E}(k, t))$, and derived a non-equilibrium scaling, written as $C_{\epsilon} \sim \operatorname{Re}_{\lambda}^{-15 / 14}$, from the evolution equation for the kinetic energy spectrum at high Reynolds numbers. This scaling is equivalent approximately to the universal law $C_{\epsilon} \sim \operatorname{Re}_{\lambda}^{-1}$, which observed in gridgenerated turbulent experiments and direct numerical simulations. In this study, the non-equilibrium $\tilde{E}(k, t)$ is considered as a harmonic wave of the equilibrium $\bar{E}(k, t)$. Accordingly, the dynamic quantities $\mathcal{U}, \mathcal{L}$ and $\epsilon$ are then all separated into their equilibrium part $\overline{\mathcal{U}}, \overline{\mathcal{L}}, \bar{\epsilon}$, and their non-equilibrium part $\tilde{\mathcal{U}}, \tilde{\mathcal{L}}, \tilde{\epsilon}$. It was shown that the large-scale quantities $\mathcal{U}$ and $\mathcal{L}$ changed considerably in the non-equilibrium procedure with $\tilde{\mathcal{L}} / \overline{\mathcal{L}} \approx \frac{3}{7} \tilde{\mathcal{U}}^{2} / \overline{\mathcal{U}}^{2}$, while the change of the small-scale quantity $\epsilon$ is negligible with $\tilde{\epsilon} / \bar{\epsilon} \approx 0$. This further confirms that the universal non-equilibrium law $C_{\epsilon} \sim \operatorname{Re}_{\lambda}^{-1}$ is intimately related to the excitation of large scales.

The non-equilibrium behaviour of $C_{\epsilon}$ is associated with the perturbed energy spectrum which can dramatically change the distribution of transfer spectrum ${ }^{[33]}$. It is, therefore, reasonable to conclude that the perturbation of energy transfer, which originate from the perturbation of energy spectrum, is the essential reason for non-equilibrium phenomena. This point of view will be explained further in Subsection 3.2. The perturbation of energy spectrum can be considered as a way in which allow the injection of perturbed transfer. This is common in turbulence closure theories, such as EDQNM model ${ }^{[61]}$ and Kovaznay model ${ }^{[62]}$, which allow the injection from energy spectrum to transfer spectrum. Besides, It is important to point out that this universal non-equilibrium dissipation process corresponds to the increasing $C_{\epsilon}$.

Now we return to discuss the relation between the evolution $S_{k}$ and the perturbation of energy spectrum. As suggested in Refs. [3], $C_{\epsilon}$ and $S_{k}$ are equivalent in describing the non-equilibrium turbulence. The experimentally results of Hearst and Lavoie also reported that $C_{\epsilon}$ and $S_{k}$ are correlated in non-equilibrium turbulent flows ${ }^{[4]}$. Therefore, the perturbation of energy spectrum can give rise to an evolution $S_{k}$ in turbulence, this can also 


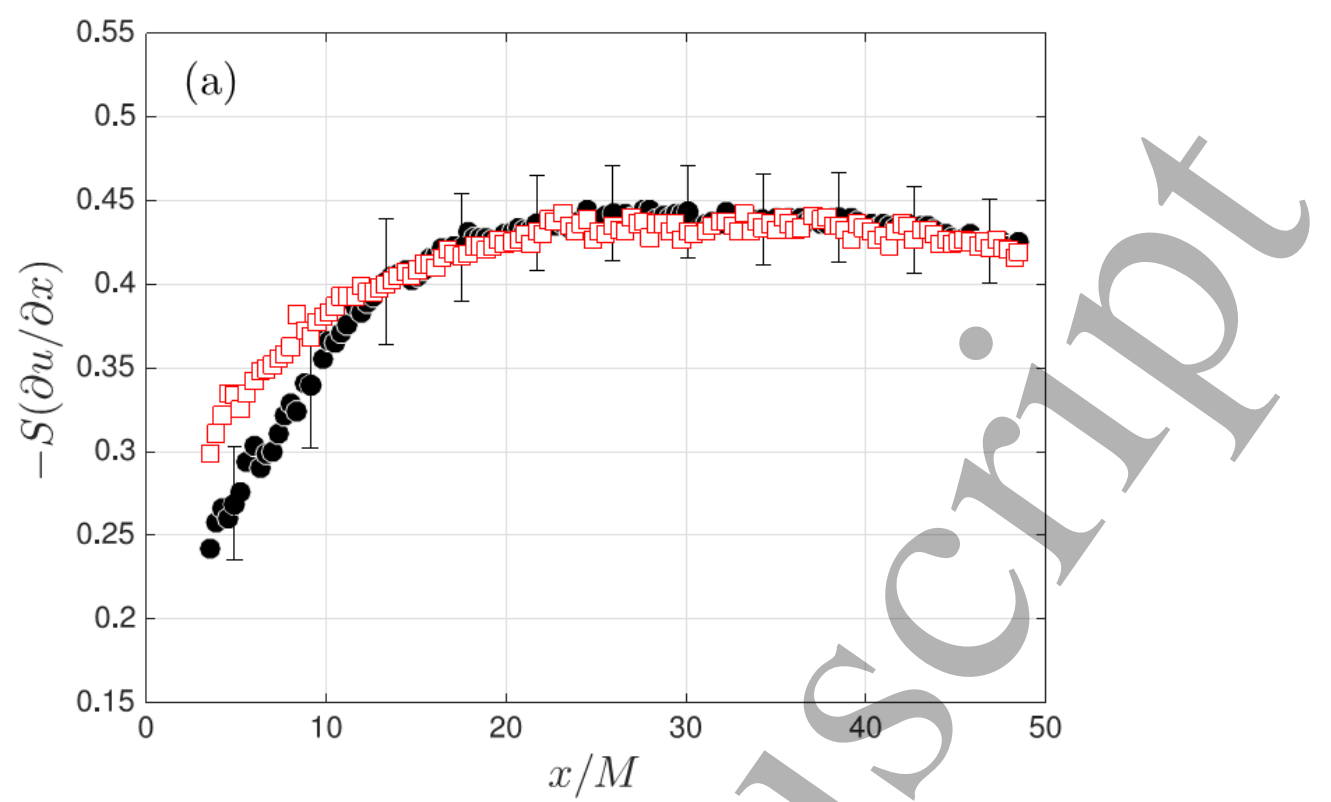

Fig. 1. Streamwise evolution of $S_{k}$ in fractal-generated grid turbulence. Different symbols correspond to different transverse locations. $S_{k}$ reaches an approximately constant at $x / M \approx 20$. Reproduced from Ref. [4], copyright 2015, with permission of AIP Publishing.

be explained with the help of equation Eq. (1). To the best of our knowledge, the existing studies on the performance of $S_{k}$ under non-equilibrium are mostly investigated in grid-generated turbulence, this relevant to the non-equilibrium near-field in which the large scales are excited by the initial generating conditions. Using fractal-generated grid turbulence, Hearst and Lavoie found that the value of $-S_{k}$ grows in the nonequilibrium near-field and then settles down to a value of around 0.43 in the equilibrium far-field (see Fig. 1) ${ }^{[4]}$. The asymptotic value of $-S_{k}$ is in line with that obtained from experimental ${ }^{[13,15,63]}$, numerical ${ }^{[7,8,10,64]}$, and theoretical investigations ${ }^{[30,65,66]}$. Isaza et al. also showed that $-S_{k}$ varies in the non-equilibrium near-field ${ }^{[5]}$, as well as the results presented by Paul et al. ${ }^{[67]}$. Introducing the concept of time-reversed turbulence, Fang et al. proposed an artificial non-equilibrium decaying turbulent case, the "Reverse-Normal" (RN), which is constructed by impose an extreme excitation suddenly on the large scales of equilibrium turbulence and keep the small scales normally in the meantime (see Refs. $[6,68]$ for more details). They found that in such case $-S_{k}$ increases in the initial stage and tends to an approximately constant after a period of time (unpublished data), according with the non-equilibrium phenomenology of the first type. Recently, large-eddy simulation (LES) for transitional spatial channel flows suggested that there is a non-equilibrium region in the later stage of transition, that is, this special region lacates before the beginning of fully developed turbulence ${ }^{[17]} .-S_{k}$ increases in this non-equilibrium region, in agreement with that of grid-generated turbulence. However, a specific character of channel flows is that the asymptotic values of $-S_{k}$ varies for different wall distances. It should be noted that $S_{k}$ remains a negative value though it changes in non-equilibrium turbulence of the first type, this agrees with that of equilibrium turbulence. A negative $S_{k}$ implies the existence of vortex stretching, which allows for a cascade from the large to the small scales in turbulence. Without a finite skewness there is no energy cascade $^{[69]}$. Generally speaking, a negative $S_{k}$ favours the formation of vortex sheets, as proposed by previous studies $\{27,38,69]$. 


\subsection{Evolution of the skewness under the perturbation of transfer spectrum}

Here we will discuss the non-equilibrium state under the perturbation of transfer spectrum. As shown by many traditional turbulence closures, the transfer spectrum is introduced by the energy spectrum, that is, the perturbation of energy spectrum in such closure can give rise to a perturbed transfer spectrum. Unfortunately, this is not the case in the present discussion, since we only want to consider the the perturbation of transfer spectrum but exclude the influence introduced by the perturbed energy spectrum. In fact, this is possible as a given energy spectrum can corresponds to multiple transfer spectra. This seems very difficult to achieve in experiments, however, it is easier in numerical simulations as proposed by the studies of Fang et al. ${ }^{[6,35,68]}$.

Reference [35] carried out a detailed analysis on the behavior of the "Reverse-Reverse"(RR) case, in which an extreme perturbation is introduced in the distribution of transfer spectrum but kept the energy spectrum in short times. They observed a new non-equilibrium law, $C_{\epsilon} \sim \operatorname{Re}_{\lambda}^{-2}$, in the short-time evolution of RR (see Fig. 2). Here we recall the main ingredients in the obtainment of this particular non-equilibrium scaling. In RR case, the perturbation is introduced at time $t=0$ of a equilibrium turbulence, and this immediately changes the transfer spectrum, from $T(k, t)$ to $-T(k, t)$, which implies the changing of energy transfer mechanism from forward to backward. It was shown that in the initial evolution stage of $\mathrm{RR}$ case $(0<t<0.2)$, the value of $C_{\epsilon}$ decreases sharply and reveals a novel dissipation scaling, $C_{\epsilon} \sim \operatorname{Re}_{\lambda}^{-2}$, this is very different from the scaling $C_{\epsilon} \sim \operatorname{Re}_{\lambda}^{-1}$ which is widely confirmed in many turbulent flows as mentioned before. In the present work, scaling $C_{\epsilon} \sim \operatorname{Re}_{\lambda}^{-2}$ is referred to as "the second non-equilibrium law". The most obvious feature of this particular non-equilibrium procedure is that the energy transfer proceeds in the backward direction, i.e., an inverse energy cascade from small to large scales. This study extends the range of traditional non-equilibrium turbulence, and more careful study needs to be done for such a new scaling law. One of the relevant discussion is introduced by the work of Bos et al. ${ }^{[54]}$, in which an initial equipartition spectrum yields the rapid decrease of $C_{\epsilon}$. Note that a backward energy transfer process is needed in their decaying case, since the energy density at the high wavenumbers is initially very high for equipartition spectrum. As a matter of fact, an inverse energy cascade exists in many turbulent flows, such as the shallow fluid layers ${ }^{[70,71]}$, the rotating turbulence ${ }^{[72-74]}$ and the corner separation region in a compressor cascade ${ }^{[75,76]}$. It is interesting that recent studies by Biferale et al. show that backward energy transfer is also available in isotropic and three-dimensional flows, and this transfer mechanism has a connection with the nature of helicity ${ }^{[77,78]}$.

The results in RR case suggest that $S_{k}$ is a positive value and decreases sharply in this novel dissipation event (see Fig. 3), indicating that a changing positive $S_{k}$ is associated with the backward energy transfer process. During this non-equilibrium procedure, the small-scale quantity $\epsilon$ evolves much faster than the largescale quantities $\mathcal{L}$ and $\mathcal{U}$, this means that the non-equilibrium of the second type is dominant by the small-scale dynamics. It should be noted that positive $S_{k}$ is very common in engineering turbulent flows, such as the transition region of channel flows ${ }^{[17]}$ and the internal flow of compressors ${ }^{[3]}$, in which the turbulence is nonequilibrium, and accompanied by strong energy backscatter. Ayyalasomayajula and Warhaft investigated the turbulent flows through an axisymmetric contraction, they found that the contraction causes a rapid increase in $S_{k}$, as the flow emerges from the contraction $S_{k}$ becomes positive, and $S_{k}$ returns to an asymptotic value of around -0.4 in the straight section of the tunnel ${ }^{[27]}$. A similar behavior is also obtained by numerous experimental $^{[25,26]}$ and numerical ${ }^{[79,80]}$ studies. Clearly, the flows through a complex geometry is universally 


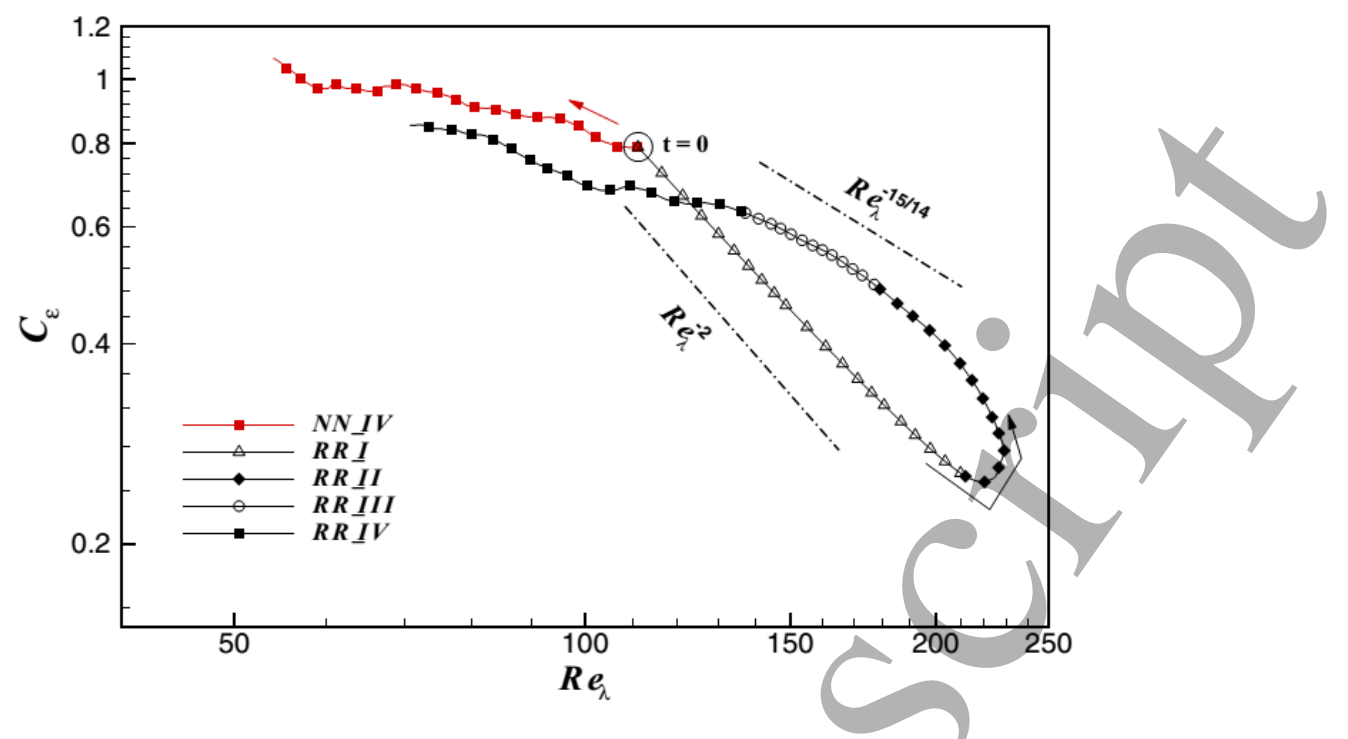

Fig. 2. $C_{\epsilon}$ versus $\mathrm{Re}_{\lambda}$ for $\mathrm{NN}$ and RR cases. NN: normally decaying case (equilibrium); RR: non-equilibrium decaying case, which is produced by reversing all velocities at time 0 . Stages $I, I I, I I I$ and $I V$ represent different time-evolution stages, among which $I$ denotes the $C_{\epsilon} \sim \operatorname{Re}_{\lambda}^{-2}$ stage, $I V$ denotes the non-equilibrium stage. Reproduced from Ref. [35], copyright 2019, with permission of American Physical Society.

non-equilibrium, and $S_{k}$ can be used to consider the energy backscatter of complex turbulence in engineering. A positive $S_{k}$ implies that vortex compression is dominating over the non-equilibrium procedure of the second type and hence the flow favours the formation of tubes rather than sheets ${ }^{[27,69]}$. This is quite different from the non-equilibrium procedure of the first type as presented in Subsection 3.1.

Since the symbols of $S_{k}$ are closely related to the structure of turbulence in non-equilibrium procedure, it is therefore necessary to track the development and evolution of turbulent structures in order to visualizing and understanding the whole process of non-equilibrium. This can be implemented with the help of the popular vortex identification methods such as Q-criterion ${ }^{[81]}, \lambda_{2}$-criterion ${ }^{[82]}, \lambda_{c i}$-criterion $^{[83,84]}, \Omega$-criterion ${ }^{[85]}$ and vortex-surface field method ${ }^{[86,87]}$, etc.

\section{The relationship between skewness and other turbulence statistics}

We focus on the relationship between skewness and enstrophy, and the relationship between skewness and turbulent kinetic energy dissipation rate in this section. Based on these analyses, it is expected to shed light on the future studies of the theoretical connection between the skewness and the non-equilibrium turbulence.

Under the assumption of isotropy, the transport equation of $\epsilon\left(\epsilon=2 \nu\left\langle s_{i j} s_{i j}\right\rangle, s_{i j}\right.$ is the fluctuating strain rate) simplifies to a form which directly related to $S_{k}{ }^{[23,88]}$, that is,

$$
-U \frac{\partial \epsilon}{\partial x}=\frac{7}{3 \sqrt{15}} \frac{\epsilon^{3 / 2}}{\nu^{1 / 2}}\left(S_{k}+2 \frac{G}{\operatorname{Re}_{\lambda}}\right),
$$

where $U$ is the mean velocity and $G=\left\langle u^{2}\right\rangle \frac{\left\langle\left(\partial^{2} u / \partial x^{2}\right)^{2}\right\rangle}{\left\langle(\partial u / \partial x)^{2}\right\rangle^{2}}$. This shows that the evolution of $\epsilon$ is intimately related to $S_{k}$. Using Eq. (2), Antoniaet al. studied the variation of $S_{k}$ in many turbulent flows ${ }^{[18,19,23,89]}$. The transport equation of $\epsilon$ has a restrictive effect on the variation of $S_{k}$, which is very important in the classic $k-\epsilon$ model. One can then simplify it by using assumptions such as local isotropy, local homogeneity, local 


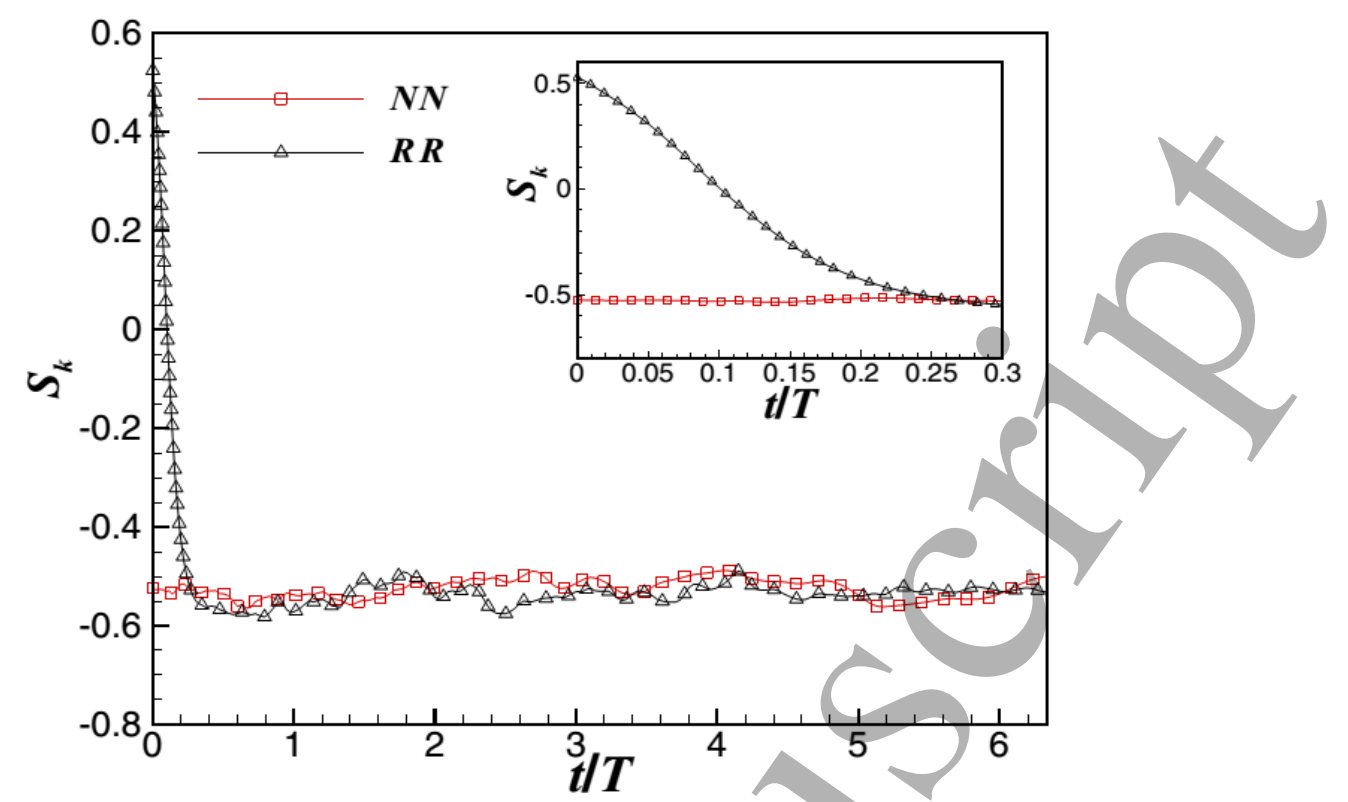

Fig. 3. Temporal evolution of $S_{k}$ for NN and RR cases. The inset is the short-time evolution of $S_{k}$. Reproduced from Ref. [35], copyright 2019, with permission of American Physical Society.

axisymmetry, and/or particular features of flow under considered. The use of the transport equation of $\epsilon$ to examine the behavior of $S_{k}$ in non-equilibrium turbulence seems to have been largely overlooked and thus it is possible to establish a theoretical relationship between $S_{k}$ and the non-equilibrium turbulence.

In addition to $\epsilon$, another statistical quantity which closely related to $S_{k}$ is the enstrophy $\frac{1}{2}\left\langle\omega_{i} \omega_{i}\right\rangle$, where $\omega_{i}$ is the vorticity of fluctuating velocity. Previous studies suggest that a negative $S_{k}$ corresponds to the generation of enstrophy via vortex stretching and a positive $S_{k}$ corresponds to the reduction of enstrophy via vortex compression ${ }^{[27,69,90]}$. This is evident in the transport equation of enstrophy ${ }^{[90]}$

$$
\frac{d}{d t}\left(\frac{1}{2}\left\langle\omega_{i} \omega_{i}\right\rangle\right)=\frac{7 \sqrt{2}}{3 \sqrt{15}} S_{k}\left(\frac{1}{2}\left\langle\omega_{i} \omega_{i}\right\rangle\right)^{3 / 2}+\nu\left\langle\omega_{i} \frac{\partial^{2} \omega_{i}}{\partial x_{j} \partial x_{j}}\right\rangle .
$$

In fact, $\epsilon$ is very similar to $\frac{1}{2}\left\langle\omega_{i} \omega_{i}\right\rangle$ as both can be regarded as statistics related to the scalar product of fluctuating velocity gradients. In addition to the turbulence production region, $\left\langle\frac{1}{2} \omega_{i} \omega_{i}\right\rangle=\left\langle s_{i j} s_{i j}\right\rangle$ in homogeneous turbulence ${ }^{[67]}$. The enstrophy production term $\left\langle\omega_{i} \omega_{j} s_{i j}\right\rangle$ appears in the transport equation of $\epsilon$ and $\frac{1}{2}\left\langle\omega_{i} \omega_{i}\right\rangle$, which further explains the fact that these two turbulence statistics are closely connected with the variation of $S_{k}$.

Although $S_{k}$ is defined under isotropic condition and has the concepts of "vertical" and "horizontal", when large-scale anisotropy is added, $S_{k}$ will be related to the direction, and thus it can be extended to anisotropic turbulence. In a recent work, we investigate the properties of $S_{k}$ in homogeneous shear turbulence which is one of the simplest case under the condition of anisotropy. $S_{k}$ is calculated along streamwise $(x)$, normal $(y)$ and spanwise $(z)$ directions, and then referred to as $S_{k}{ }^{x}, S_{k}{ }^{y}$, and $S_{k}{ }^{z}$ respectively. It is shown that the values of $S_{k}{ }^{x}$, $S_{k}{ }^{y}$ and $S_{k}{ }^{z}$ are distinct, while they trend together after a filter operation. The filter can change the small-scale information but has no effect on large scales. This means that the small-scale quantity $S_{k}$ is markedly directional in anisotropic turbulence. The directional character disappeared gradually as some small-scale information is filtered. Fang et al. made an attempt to investigate the property of anisotropy by the scaling of the second-order 
cross structure function ${ }^{[91]}$. However, $S_{k}$ is assumed under isotropic condition, which limits the application of this theory. If the directional character is taken into account, it is expected to develop a credible model for $S_{k}$ under anisotropic conditions. The progress of subgrid-scale modeling in physical space and the understanding on the mathematical constraints in the modeling process are helpful in modeling the $S_{k}{ }^{[92,93]}$. Further studies are therefore needed in this respect. In a word, it is expected to explore the non-equilibrium mechanism of anisotropic turbulence by investigating the relationship between $S_{k}$ and $\frac{1}{2}\left\langle\omega_{i} \omega_{i}\right\rangle$ and the relationship between $S_{k}$ and $\epsilon$.

\section{Conclusions and perspectives}

In this paper, we present a comprehensive review on the connections between $S_{k}$ and the non-equilibrium evolution of turbulence. $S_{k}$ varies in the non-equilibrium turbulence. The changing negative $S_{k}$ corresponds to a non-equilibrium procedure of the first type, in which the turbulent kinetic energy decay rapidly; while the changing positive $S_{k}$ corresponds to a non-equilibrium procedure of the second type, in which the energy transfer proceeds in the backward direction. The perturbation of energy spectrum is the origin of non-equilibrium of the first type, by contrast, the non-equilibrium of the second type is excited by the perturbation of transfer spectrum. Besides, the transport equations of turbulent energy dissipation rate and enstrophy have a restrictive effect on the variation of $S_{k}$.

However, most of the existing researches focus on observing the variation of $S_{k}$, and its theoretical relationship with non-equilibrium turbulence has not been established. Specifically, the future studies should be focused on the following aspects:

1. Previous studies have focused mainly on the non-equilibrium turbulence which excited by the perturbation of energy spectrum or transfer spectrum in a certain wavenumber range, what happens to the value of $S_{k}$ when the excitation is imposed in a specific wavenumber.

2. By analysing the transport equation of turbulent energy dissipation rate and enstrophy, it might be possible to establish a theoretical connection between skewness and the non-equilibrium turbulence, this then calls for future studies.

3. $S_{k}$ is a promising candidate for investigating the physical mechanism of non-equilibrium under anisotropy, thus how to introduce the anisotropy to the non-equilibrium turbulence is an important problem.

4. Are there any other non-equilibrium procedures of different type in addition to the two which mentioned in this paper? In non-equilibrium turbulence, what law does $S_{k}$ follow and how turbulent structures evolve?

The present work is expected to inspire future investigations for the turbulent flow mechanism under complex conditions as well as the modelling of non-equilibrium turbulence.

\section{References}

1] Vạssilicos J C 2015 Annu. Rev. Fluid Mech. 4795

[2] Castro I P 2016 J. Fluid Mech. 7881 
[3] Fang L, Zhao H K, Lu L P, Liu Y W and Yan H 2017 Aerosp. Sci. Technol. 7178

[4] Hearst R J and Lavoie P 2015 Phys. Fluids 27071701

[5] Isaza J C, Salazar R and Warhaft Z 2014 J. Fluid Mech. 753402

[6] Fang L, Zhu Y, Liu Y W and Lu L P 2015 Phys. Lett. A 3792331

[7] Ishihara T, Kaneda Y, Yokokawa M, Itakura K and Uno A 2007 J. Fluid Mech. 592335

[8] Wang L P, Chen S Y, Brasseur J G and Wyngaard J C 1996 J. Fluid Mech. 309113

[9] Sreenivasan K R and Antonia R A 1997 Annu. Rev. Fluid Mech. 29435

[10] Gotoh T, Fukayama D and Nakano T 2002 Phys. Fluids 141065

[11] Hill R J 2002a J. Fluid Mech. 452361

[12] Gylfason A, Ayyalasomayajula S and Warhaft Z 2004 J. Fluid Mech. 501213

[13] Burattini P, Lavoie P and Antonia R A 2008 Exp. Fluids 45523

[14] Tabeling P, Zocchi G, Belin F, Maurer J and Willaime H 1996 Phys. Rev. E 531613

[15] Belin F, Maurer J, Tabeling P and Willaime H 1997 Phys. Fluids 93843

[16] Atta C W V and Antonia R A 1980 Phys. Fluids 23252

[17] Liu F, Lu L P and Fang L 2018 J. Turbul. 19731

[18] Thiesset F, Antonia R A and Djenidi L 2014 J. Fluid Mech. 748 R2

[19] Tang S L, Antonia R A, Djenidi L and Zhou Y 2015 J. Fluid Mech. 784109

[20] Lavoie P, Djenidi L and Antonia R A 2007 J. Fluid Mech. 585395

[21] Lee S K, Djenidi L, Antonia R A and Danaila L 2013 Int. J. Heat/Fluid Flow 43129

[22] Tatsumi T, Kida S and Mizushima J 1978 J. Fluid Mech. 8597

[23] Antonia R A, Tang S L, Djenidi L and Danaila L 2015 J. Fluid Mech. 781727

[24] Djenidi L, Antonia R A, Talluru M K and Abe H 2017 Phys. Fluids 2064608

[25] Mills R R and Corrsin S 1959 NASA-MEMO $\mathbf{1 9 9 8 0 2 2 8 0 3 1}$

[26] Sjogren T and Johansson A V 1998 J. Fluid Mech. 37459

[27] Ayyalasomayajula S and Warhaft Z 2006/J. Fluid Mech. 566273307

[28] Bos W J T, Chevillard L, Scott J and Rubinstein R 2012 Phys. Fluids 24015108

[29] Tavoularis S, Bennett J C and Corrsin S 1978 J. Fluid Mech. 8863

[30] Qian J 1994 Acta Mech. Sin. 1012

[31] Nelkin M 1990 Phys. Rev. A 427226

[32] Goto S and Vassilicos J C 2016 Phys. Rev. E 94053108

[33] Meldi M and Sagaut P 2018 J. Turbul. 19390

[34] Takamure K, Sakai Y, Ito Y, Iwano K and Hayase T 2019 Int. J. Heat Fluid Flow 757785

[35] Liu F, Lu L P, Bos W J T and Fang L 2019 Phys Rev Fluids 4084603

[36] Deissler R G 1978 Appl. Sci. Res. 34379

[37] Kolmogorov A N 1941 Dokl. Akad. Nauk. SSSR 3216

[38] Batchelor G K 1953 The theory of homogeneous turbulence (Cambridge University Press)

[39] Waleffe F 1992 Phys. Fluids A 4350

[40] Yakhot V and Orszag S A 1986 J. Sci. Comput. 13

[41] Domaradzki J A and Rogallo R S 1990 Phys. Fluids A 2413

[42] Valente P C, Onishi R and da Silva C B 2014 Phys. Rev. E 90023003

[43] Goto S and Vassilicos J C 2015 Phys. Lett. A 3791144

[44] Seoud R E and Vassilicos J C 2007 Phy. Fluids 19105108

[45] Valente P C and Vassilicos J C 2014 J. Fluid Mech. 7445

[46] Valente P C and Vassilicos J C 2012 Phys. Rev. Lett. 108214503

[47] Mazellier N and Vassilicos J C 2008 Phy. Fluids 20015101

[48] Nedic J, Vassilicos J C and Ganapathisubramani B 2013 Phys. Rev. Lett. 111144503

[49] Dairay T, Obligado M and Vassilicos J C 2015 J. Fluid Mech. 781166 
[50] Obligado M, Dairay T and Vassilicos J C 2016 Phy. Fluids 1044409

[51] Layek G C and Sunita 2018 Phys Rev Fluids 3124605

[52] Layek G C and Sunita 2018 Phy. Fluids 30115105

[53] Nedic J, Tavoularis S and Marusic I 2017 Phys Rev Fluids 2032601

[54] Bos W J T, Shao L and Bertoglio J P 2007 Phy. Fluids 19045101

[55] Davies P O A L and Yule A J 1975 J. Fluid Mech. 69513537

[56] Melina G, Bruce P J K and Vassilicos J C 2016 Phys Rev Fluids 1044402

[57] Wiltse J M and Glezer A 1998 Phy. Fluids 102026

[58] Wiltse J M and Glezer A 1993 J. Fluid Mech. 249261

[59] Yeung P K, Brasseur J G and Wang Q 1995 J. Fluid Mech. 28343

[60] Bos W J T and Rubinstein R 2017 Phys Rev Fluids 2022601

[61] Orszag S A 1970 J. Fluid Mech. 41363

[62] Kovaznay L S G 1948 J. Aeron. Sci. 15745

[63] Frisch U 1995 Turbulence: The legacy of A.N. Kolmogorov (Cambridge University Press)

[64] Orszag S A and Patterson G S 1972 Phys. Rev. Lett. 2876

[65] Andre J C and Lesieur M 1977 J. Fluid Mech. 81187

[66] Kraichnan R H 1990 Phys. Rev. Lett. 65575

[67] Paul I, Papadakis G and Vassilicos J C 2017 J. Fluid Mech. 815295

[68] Fang L, Bos W J T, Shao L and Bertoglio J P 2012 J. Turbul. 13 1

[69] Davidson P A 2004 Turbulence: An Introduction for Scientists and Engineers (Oxford University Press)

[70] Nastrom G D, Gage K S and Jasperson W H 1984 Nature 31036

[71] Celani A, Musacchio S and Vincenzi D 2010 Phys. Rev. Lett. 104184506

[72] Jacobitz F G, Schneider K, Bos W J T and Farge M 2010 Phy. Fluids 22085101

[73] Yarom E, Vardi Y and Sharon E 2013 Phy. Fluids 25085105

[74] Campagne A, Gallet B, Moisy F and Cortet P P 2014 Phy. Fluids 26125112

[75] Liu Y W, Lu L P, Fang L and Gao F 2011 Phys. Lett. A 3752377

[76] Yan H, Liu Y W, Li Q S and Lu L P 2018 Aerosp. Sci. Technol. 75139

[77] Biferale L, Musacchio S and Toschi F 2012 Phys. Rev. Lett. 108164501

[78] Sahoo G, Alexakis A and Biferale L 2017 Phys. Rev. Lett. 118164501

[79] Lee C M, Gylfason A, Perlekar P and Toschi F 2015 J. Fluid Mech. 78531

[80] Clay M P and Yeung P K 2016 J. Fluid Mech. 805460

[81] Hunt J C R, Wray A and Moin P 1988 In Center for Turbulence Research Report CTR-S88 193

[82] Jeong J and Hussain F 1995 J. Fluid Mech. 28569

[83] Berdahl C and Thompson D 1993 AIAA J. 3197

[84] Zhou J, Adrian R J, Balachandar S and Kendal T M 1999 J. Fluid Mech. 387353

[85] Liu C Q, Wang Y Q, Yang Y and Duan Z W 2016 Sci. China-Phys. Mech. Astron. 59684711

[86] Yang Y and Pullin D I 2010 J. Fluid Mech. 661446

[87] Xiong S and Yang Y 2019 J. Fluid Mech. 874952

[88] Batchelor G K and Townsend A A 1947 Proc. R. Soc. London, Ser. A 190534

[89] Tang S L, Antonia R A, Djenidi L, Abe H, Zhou T, Danaila L and Zhou Y 2015 J. Fluid Mech. 777151

[90] Lesieur M 1997 Turbulence in Fluids (Kluwer Academic, Dordrecht)

[91] Fang L and Wang L P 2015 Ninth International Symposium on Turbulence and Shear Flow Phenomena, June 30-July 3, 2015, Melbourne, Australia

[92] Fang L, Shao L and Bertoglio J P 2014 Sci. China-Phys. Mech. Astron. 572188

[93] Fang L and Ge M W 2017 Chinese Phys. Lett. 34030501 